\title{
Historical Supernovae and their Remnants
}

\author{
D.A. Green \\ Mullard Radio Astronomy Observatory, Cavendish Laboratory, \\ Madingley Road, Cambridge CB3 OHE, U.K.
}

\begin{abstract}
Historical records are available for nine supernova explosions seen in our Galaxy over the last two millennia. The observations of these 'historical' supernovae are very useful for the modern astrophysical interpretation of the remnants of these supernovae. The ages of the remnants of these historical supernovae are definitively known, which greatly aids understanding of the nature of the remnants. Also, the fact that the parent supernova was seen implies that it was relatively nearby in the Galaxy, giving further constraints on the distance, and hence other properties of their remnants. Here I briefly review the historical supernovae in our Galaxy, in the context of our understanding of their remnants from modern observations.
\end{abstract}

\section{Introduction}

This paper reviews Galactic Supernovae (SN) for which historical observations are available to us, and their remnants. The properties of SN and supernova remnants (SNRs) are briefly outlined in Section 2, and the 'historical' SN particularly two examples: the SN of around AD 1680 with produced Cas A, and the SN of AD 1181 and its remnant 3C58 - are discussed in Section 3. Finally, other young SNRs in the Galaxy not seen historically are briefly discussed in Section 4 .

\section{Background}

\subsection{Supernovae}

Simplistically there are two types of Supernovae observed: 'type I' SN which do not show hydrogen in optical spectra near maximum, and are seen in both elliptical and spiral galaxies; and 'type II' SN which do show hydrogen in optical spectra near maximum, and are seen in spiral galaxies only. From these observed properties of SN it is deduced that 'type I' SN are from less massive stars, and 'type II' SN from more massive stars. In each type of SN a lot of mass and energy $-\sim 10^{44} \mathrm{~J}-$ is released, which interacts with the surrounding interstellar medium to produce extended Supernova Remnants (SNRs). Also, some of 'type II' SN are expected to leave behind a compact remnant (e.g. a neutron star). About one SN per 50 years is expected in our Galaxy, although most will not be seen optically, due to absorption. 


\subsection{Supernova Remnants (SNRs)}

Of the $\sim 225$ known Galactic SNRs (Green 2000), almost all are detected at radio wavelengths, with only about $30 \%$ detected in X-rays, and $20 \%$ optically. SNRs are classified into three types:

1. $\sim 85 \%$ are 'shell' type SNRs - like the remnant of Tycho's SN AD1572 showing more or less complete limb-brightened rings of emission;

2. $\sim 5 \%$ are 'filled-centre' (or 'plerions') - like the Crab Nebula, which is the remnant of the SN of AD 1054 - showing centrally brightened emission (although it should be noted that the Crab is not typical of this class of SNRs);

3. $\sim 10 \%$ are 'composite' types, showing properties of both 'shell' and 'filledcentre' remnants.

SNRs are important as energy source for heating the ISM, and thought to be the source of cosmic ray acceleration (at least up to $\sim 10^{15} \mathrm{eV}$ ).

\section{The Historical Supernovae}

Details of the known historical SN are given in Table 1 (see, for example, Clark \& Stephenson 1977; Ashworth 1980). For the SN of $\mathrm{AD} 1680$ ? there is only one European record (see sub-section 3.1), and for the SNe of AD 1604 and AD 1572, although oriental records do exist, it is the European records that provide us with the best information on the position and brightness of the SN. For earlier SN, it is almost only from oriental records that we have details of these $\mathrm{SNe}$, although for the probable SNe of AD 393, AD 385 and AD 185 there are only limited, and terse records available, so that the properties of the SN are uncertain, and hence there is uncertainty about their remnants. It should be noted that the type of the SN cannot be deduced with confidence from the historical records.

Table 1. The Historical Supernovae and their Remnants.

\begin{tabular}{cccc}
\hline Year & duration & magnitude & remnant \\
\hline AD 1680? & - & $?$ & Cas A \\
AD 1604 & 12 months & -2.5 & Kepler's SNR \\
AD 1572 & 6 months & -4 & Tycho's SNR \\
AD 1181 & 6 months & -1 & 3C58 \\
AD 1054 & 22 months & -4 & Crab Nebula \\
AD 1006 & several years & -9 & G327.6+14.5 \\
AD 393? & 8 months & $-1 ?$ & CTB37A or B? \\
AD 385? & 3 months & $+1 ?$ & G11.2-0.3? \\
AD 185? & 20 months & $-8 ?$ & G315.4-2.5 or G320.4-1.2? \\
\hline \hline
\end{tabular}




\subsection{The SN of around $A D 1680$ and Cas $A$}

The SNR Cassiopeia A (Cas A, $=3 \mathrm{C} 461,=\mathrm{G} 111.7-2.1$ ), is among the brightest radio sources in the sky, and is a 'limb-brightened' radio shell $\approx 5$ arcmin in diameter, with many, expanding bright knots (see Anderson \& Rudnick 1995). Cas A has faint optical filaments and knots, spectra showing that this is ejecta from a massive star (i.e. its parent SN was a 'type II'). In X-rays, Cas A has a bright shell, with a recently identified central compact source (Tananbaum et al. 1999), thought to be a neutron star (e.g. Umeda et al. 2000). The SN that produced Cas A was apparently seen by Flamsteed in AD1680, although it was not recognised as an usual or new star at the time, as it was apparently not very bright. The historical identification of this SN is important for studies of Cas $\mathrm{A}$, as it provides a definitive age for the remnant which is important for the expansion studies of SNR, and for cooling models for neutron stars.

\subsection{The SN of AD 1181 and $3 C 58$}

The SN of AD 1181 was reported in the northern and southern Chinese empires, and in Japan (see Stephenson \& Green 1999), but there are no European records of this SN. The following record from South China - from the Wen-hsien T'ung $k$ 'ao - gives the approximate position the 'guest star', and clearly states that this was seen for a long time (therefore excluding the possibility that this was a report of a shorter-lived phenomenon, such as a nova or comet).

"Ch'un-hsi reign period (of emperor Hsiao-tsung), year 8, month VI, day chi-szu (AD 1181 Aug 6). A guest star appeared in K'uei lunar lodge, invading (fan) the stars of Ch'uan-she until the following year, month I, day kuei-yu (AD 1182 Feb 6), a total of 185 days. Only then was it extinguished (mieh)"

Although the position of the SN was not very well defined (a few degrees only), the identification of its remnant, which was first made in 1971, is now firmly with the radio source $3 \mathrm{C} 58(=\mathrm{G} 130.7+3.1)$. This is a centrally brightened (or "filled-centre") radio source $\approx 5 \times 10 \mathrm{arcmin}^{2}$ in extent (e.g. Green 1986), which has faint optical filaments, and shows centrally brightened X-ray emission. Xray observations also show that $3 \mathrm{C} 58$ contains a compact source, as is expected to explain the remnant's centrally brightened morphology (Helfand, Becker, \& White 1995). The presence of the centre compact source implies that the SN of AD 1181 was a 'type II'.

The integrated spectrum of 3C58 shows a sharp break somewhere above $\sim$ $100 \mathrm{GHz}$ (see Green \& Scheuer 1992), which is at a considerably lower frequency than the break seen in the Crab Nebula (which is the remnant of the SN of $\mathrm{AD} 1054$, and is therefore of similar age to 3C58). This difference in the spectral shape may provide an important clue as to the emission mechanism at work in filled-centre remnants, and the interpretation of this depends on knowing the age of this remnant from the historical observations.

\section{Other young SNRs in the Galaxy?}

The remnants of the historical SN are all relatively close by (within $\sim 5 \mathrm{kpc}$ ) - as is expected since their parent $\mathrm{SNe}$ were seen optically - and consequently there 
should be tens other young, but distant, SNRs in the Galaxy. Although there are some other young (small, high surface brightness) SNRs in current catalogues, most are missing, due to observational selection effects (see, for example, Green 1991, and references therein).

In addition, there may be other nearby $\mathrm{SN}$ for which no historical records have yet been identified. For example, the recently identified SNR G266.2-1.2 in Vela (Aschenbach 1998; Duncan \& Green 2000) may be only around seven hundred years old, and is close by, so that historical records might be expected. (It has even been proposed that evidence for this young SN may be visible in nitrate concentrations in Antarctic ice cores, see Burgess \& Zuber 2000)

\section{Conclusions}

The historical records of SN are not only of interest in themselves, but are useful for astrophysical studies of their remnants. Additional records of the already known historical SN may be useful for studies of the individual remnants, and records of additional historical SN would be particularly useful to provide definitive ages for other SNRs.

\section{References}

Anderson, M.C., \& Rudnick, L. 1995, ApJ, 441, 307

Aschenbach, B. 1998, Nature, 396, 141

Ashworth, W.B. 1980, JHA, 11, 1

Burgess, C.P., \& Zuber, K. 2000, APh, 14, 1

Clark, D.H., \& Stephenson, F.R. 1977, The Historical Supernovae, (Oxford: Pergamon)

Duncan, A.R., \& Green, D.A. 2000, A\&A, in press

Green, D.A. 1986, MNRAS, 218, 533

Green, D.A. 1991, PASP, 103, 209

Green, D.A. 2000, "A Catalogue of Galactic Supernova Remnants (2000 August version)", Mullard Radio Astronomy Observatory, Cavendish Laboratory, Cambridge, United Kingdom (available on the World-Wide-Web at "http://www.mrao.cam.ac.uk/surveys/snrs/")

Green, D.A. \& Scheuer, P.A.G. 1992, MNRAS, 258, 833

Helfand, D.J., Becker, R.H., \& White, R.L. 1995, ApJ, 453, 741

Stephenson, F.R., \& Green, D.A. 1999, A\&G, 40, 27

Tananbaum, H. et al. 1999, IAUC No. 7246

Umeda, H. Nomoto, K. Tsuruta, S., \& Mineshige, S. 2000, ApJ, 534, L193 